\title{
Hopf term and the effective Lagrangian for the Skyrmions in a two-dimensional electron gas at small $g$-factor
}

\author{
W. Apel ${ }^{1}$ and Yu. A. Bychkov ${ }^{2,1}$ \\ 1 Physikalisch-Technische Bundesanstalt, Bundesallee 100, 38116 Braunschweig, Germany. \\ ${ }^{2}$ L.D. Landau Institute for Theoretical Physics, ul.Kosigina, 2, Moscow, Russia.
}

(July 6, 2021)

\begin{abstract}
We study interacting electrons in two dimensions moving in the lowest Landau level under the condition that the Zeeman energy is much smaller than the Coulomb energy and the filling factor is one. In this case, Skyrmion quasiparticles play an important role. Here, we present a simple and transparent derivation of the corresponding effective Lagrangian. In its kinetic part, we find a non-zero Hopf term the prefactor of which we determine rigorously. In the Hamiltonian part, we calculate, by means of a gradient expansion, the SkyrmionSkyrmion interaction completely up to fourth order in spatial derivatives.
\end{abstract}

PACS numbers: 73.20.Dx, 73.20.Mf, 71.35.Ji, 75.30.Et

Two-dimensional electron gases as manufactured in GaAs heterostructures show a rich variety of features as the strength of a magnetic field or the particle density is varied. Most prominent among these are the quantum Hall effects (QHEs) at integer and fractional filling factors $\nu\left(\nu=N_{e} / N_{\Phi}\right.$, where $N_{e}$ and $N_{\Phi}$ are the number of electrons and the orbital degeneracy of the Landau level, respectively). The existence of a well-defined singleparticle spectrum of discrete, spin-split Landau levels allows one to explain the integer QHE. Until recently it was accepted that when in such a system the characteristic Coulomb energy is less than the cyclotron energy, the ordinary low-lying excitations are electron-hole pairs of opposite spins (spin excitons [1,2]). These have a nonzero kinetic energy with a strong $k$-dispersion due to the electron-electron interaction. In a completely filled Landau level, the energy gap for creating a widely separated quasielectron-quasihole pair, a large spin exciton (i.e. one with $k \rightarrow \infty$ ), is apart from the Zeeman splitting governed by the exchange energy associated with a hole, and is equal to $|g| \mu_{B} B+\sqrt{\frac{\pi}{2}} \frac{e^{2}}{\kappa l_{H}}$. Here, $g$ is the effective single particle Landé factor and $l_{H}=(c \hbar / e B)^{1 / 2}$ is the magnetic length. This is in qualitative agreement with experiments on the temperature-dependent longitudinal resistance in the thermally activated regime 3, 1,

Recent theoretical investigations [5,6] near filling factor $\nu=1$, however, revealed that the interplay between Zeeman and Coulomb interaction results in a more complex type of excitations with unusual spin order which can be described as Skyrmions. It was shown [5] that the energy gap required to create a widely separated Skyrmion-antiSkyrmion pair, is only half of the gap required to create a large spin exciton. Skyrmions appeared originally in Condensed Matter physics as solutions of the $O(3)$ nonlinear sigma model in two dimensions for non-zero values of the topological charge [7]. Provided now that the Zeeman energy is less than the Coulomb energy, the electron gas is equivalent to an isotropic itinerant ferromagnet. The latter can then be described by a three-component order parameter in a $2 \mathrm{D}$ coordinate space, i.e. just by the $O(3)$ non-linear sigma model [7 9] in which one finds topologically non-trivial spin-textures, the Skyrmions. Evidence for a realization of these unusual excitations in a $2 \mathrm{D}$ electron gas under a strong magnetic field has recently been uncovered in a number of experimental papers 10 13 which use quite different techniques.

Recently, there has also been a lot of theoretical work dealing with the case of filling factor $\nu$ near and at 1 , i.e. with two spin-split Landau sub-levels; this case is of interest here. In the problem of a double-layer system, intimately related to our case, several derivations of a Lagrangian for the Skyrmions have been discussed in [14]. For finite $|\nu-1|$, a periodic arrangement, a crystal of Skyrmions was studied [15]. Starting from a Lagrangian description, Ref. 16] discussed finite temperature properties within a scaling theory. The many body perturbation theory approach was employed in [17] to study the thermodynamics. A different line was followed by one of the present authors 18]. It builds on the observation that the Hartree-Fock (HF) approximation is valid for small $g$-factor, when the Skyrmions contain many reversed spins. Then, the HF state is parameterized by a unit vector $\vec{n}(\vec{r})$ related to the spin-density. The energy is invariant against a uniform rotation of $\vec{n}$. Expanding the HF energy in spatial derivatives leads to a $O(3)$ nonlinear sigma model with a topological term (describing the map $S_{2} \rightarrow S_{2}$ ). Here, the latter has a real prefactor, in remarkable contrast to other cases of physical realizations of this model, e.g. the Heisenberg chain where an imaginary prefactor describes the difference between integer and half-integer spin cf. 19.9.9.

There are two very important problems connected with the physics of Skyrmions. The first regards the interaction between the Skyrmions. In order to describe this properly, the gradient expansion of the energy functional [18 valid in the case of a very small Zeeman energy should be continued at least up to the fourth order in 
derivatives. The second problem lies in the dynamics of the Skyrmions and their spin and statistics. These are determined in the Lagrangian by the terms containing time derivatives. Now the main theoretical question is how to derive the corresponding macroscopic Lagrangian from the microscopic Hamiltonian of interacting electrons in two spin-split Landau sub-levels. The particular challenge there regards the quantum nature of the Skyrmion excitations. It is determined by the "Hopf term" [20,9] which is topological in nature and, obviously, cannot be found by a simple generalization of the classical model but rather needs to be derived from the microscopic Hamiltonian of the electrons. This constitutes the objective of the present work. So far, it was unclear whether the prefactor of a Hopf term in the action is non-zero and how it could be found from microscopic calculations. In this Letter, we set out to give a complete derivation of all parts of the effective action up to and including the fourth order in derivatives. This is not a trivial task, since we are expecting terms which are total derivatives as the Hopf term [21] and thus can be easily overlooked. Our treatment reveals the full $S U(2)$ symmetry of the problem which is displayed by the kinetic part of the action. The Hamiltonian part, on the other hand, shows $S U(2) / U(1)$ symmetry. With our result, the statistics and the interaction of the Skyrmions are then rigorously determined. As far as we are aware, this is the first case in Condensed Matter theory in which one can calculate a non-zero Hopf term from the microscopic model and thus determine the statistics of the quasiparticles.

We study interacting electrons in the lowest Landau level. Using the Landau gauge, the destruction operator of a spin up (down) electron with linear momentum $p$ is denoted by $\hat{a}_{p}\left(\hat{b}_{p}\right)$. Then, the Hamiltonian $\hat{H}$ reads

$$
\begin{gathered}
\hat{H}=\frac{1}{2} \sum_{\vec{q}, p_{1}, p_{2}} \tilde{V}(q) e^{i q_{x}\left(p_{2}^{\prime}-p_{1}\right)}\left[\hat{a}_{p_{1}}^{\dagger} \hat{a}_{p_{2}}^{\dagger} \hat{a}_{p_{2}^{\prime}} \hat{a}_{p_{1}^{\prime}}+(\hat{a} \rightarrow \hat{b})\right. \\
\left.+2 \hat{a}_{p_{1}}^{\dagger} \hat{b}_{p_{2}}^{\dagger} \hat{b}_{p_{2}^{\prime}} \hat{a}_{p_{1}^{\prime}}\right]
\end{gathered}
$$

Here, as in [18], $\tilde{V}(q)=e^{-q^{2} / 2} V(q)$, where $V(q)$ is the electron-electron interaction, and $p_{1}^{\prime}=p_{1}-q_{y}, p_{2}^{\prime}=$ $p_{2}+q_{y}$. All lengths are measured here in units of magnetic length $l_{H}$. We write the HF state as $\left|\Psi_{H F}>=\Pi_{p} \hat{A}_{p}^{\dagger}\right| 0>$ (denoted by $\mid \psi>$ in [18]) where the creation operators $\hat{A}_{p}^{\dagger}$ of the single particle HF states are given by

$$
\hat{A}_{p}=\sum_{p_{1}}\left(U_{p, p_{1}} \hat{a}_{p_{1}}+V_{p, p_{1}} \hat{b}_{p_{1}}\right)
$$

with

$$
\hat{U}=e^{i \hat{\psi} / 2} \cos \frac{\hat{\theta}}{2} e^{i \hat{\phi} / 2}, \quad \hat{V}=e^{i \hat{\psi} / 2} \sin \frac{\hat{\theta}}{2} e^{-i \hat{\phi} / 2} .
$$

The matrices $\hat{U}$ and $\hat{V}$ represent a transformation of $\hat{a}_{p}$ and $\hat{b}_{p}$ which respect the anticommutation rules. They are parameterized by three Euler angles, $\psi(\vec{r}), \theta(\vec{r})$, and $\phi(\vec{r})$ as follows. The elements $\psi_{p, p^{\prime}}$ of the matrix $\hat{\psi}$ are to be calculated with the Landau states from $\psi(\vec{r})$ as $\psi_{p, p^{\prime}}=[\psi(\vec{r})]_{p, p^{\prime}}$, and correspondingly for $\hat{\theta}$ and $\hat{\phi}$. In all the calculations below, we shall use the techniques developed in [18]. For each matrix $\hat{M}$, define a corresponding function

$$
\tilde{M}(\vec{q})=\sum_{p} e^{i q_{x}\left(p+q_{y} / 2\right)} M_{p+q_{y}, p} .
$$

Then, e.g.

$\tilde{\theta}(\vec{q})=e^{-\frac{1}{4} q^{2}} \int \frac{d^{2} r}{2 \pi} \theta(\vec{r}) e^{-i \vec{q} \cdot \vec{r}}=: \int \frac{d^{2} r}{2 \pi} \bar{\theta}(\vec{r}) e^{-i \vec{q} \cdot \vec{r}}$.

$\bar{\theta}(\vec{r})$ differs from $\theta(\vec{r})$ in that it is averaged over the area of one flux quantum. Since we are interested in a gradient expansion in which functions vary slowly on the scale of the magnetic length, the renormalized $\bar{\theta}$ is the natural variable in which we expect to express our results. In the following, we repeatedly need functions of matrices as in (3). With the definition (4) and using the composition rule Eq. (20) in 18 we get up to fourth order in gradients e.g.

$$
\int \frac{d^{2} q}{2 \pi} e^{i \vec{q} \cdot \vec{r}}\left[\cos \frac{\hat{\theta}}{2}\right](\vec{q})=\left(1+\mathcal{O}\left(\nabla^{4}\right)\right) \cos \frac{\bar{\theta}(\vec{r})}{2}
$$

and similarly for the other functions in (3).

We now proceed with our derivation of the effective Lagrangian. The HF approximation of $\hat{H}$ reads 18$]$

$$
\begin{aligned}
<\hat{H}>=\sum_{\vec{q}}\{ & \frac{1}{2}[\tilde{V}(q)-\pi E(q)] \tilde{N}(\vec{q}) \tilde{N}(-\vec{q}) \\
& \left.-2 \pi E(q) \sum_{j=x, y, z} \tilde{S}^{j}(\vec{q}) \tilde{S}^{j}(-\vec{q})\right\} .
\end{aligned}
$$

Here, $E(q) \equiv \int \frac{d^{2} p}{(2 \pi)^{2}} \tilde{V}(p) e^{i \vec{p} \cdot \vec{q}}$, and the matrix corresponding to the HF expectation value of the chargedensity $\tilde{N}(\vec{q})$ is given by

$$
\hat{N}=\hat{U}^{\dagger} \hat{U}+\hat{V}^{\dagger} \hat{V},
$$

while the matrices corresponding to the HF expectation values of the vector components of the spin-density $\tilde{S}^{j}(\vec{q})$ are

$$
\hat{S}^{x}+i \hat{S}^{y}=\hat{V}^{\dagger} \hat{U} \quad, \quad \hat{S}^{z}=\frac{1}{2}\left[\hat{U}^{\dagger} \hat{U}-\hat{V}^{\dagger} \hat{V}\right] .
$$

$\hat{N}$ and $\hat{S}^{j}$ and thus (7) do not involve the angle $\bar{\psi}$ which, therefore, was not taken into account in $[18]$. Here, it will be necessary to keep the transformation as general as in (3) as will become clear in the sequel.

After having defined the Hamiltonian and the HF state, proceeding now to evaluating the effective action, we consider time dependent HF states $\mid \Psi_{H F}>$ parameterized 
by $\bar{\psi}(\vec{r}, t), \bar{\theta}(\vec{r}, t)$, and $\bar{\phi}(\vec{r}, t)$. Then, our task is to expand the effective Lagrangian (real time)

$$
\mathcal{L}=<\Psi_{H F}\left|i \partial_{t}\right| \Psi_{H F}>-<\Psi_{H F}|\hat{H}| \Psi_{H F}>
$$

with respect to gradients in the fields $\bar{\psi}, \bar{\theta}$, and $\bar{\phi}$ up to and including fourth order.

Kinetic part In calculating

$$
\begin{aligned}
\mathcal{L}_{k} & =<\Psi_{H F}\left|i \partial_{t}\right| \Psi_{H F}>=\sum_{p}<0\left|\hat{A}_{p} i \partial_{t} \hat{A}_{p}^{\dagger}\right| 0> \\
& =\sum_{p, p_{1}}\left\{U_{p, p_{1}} i \partial_{t} U_{p_{1}, p}^{\dagger}+V_{p, p_{1}} i \partial_{t} V_{p_{1}, p}^{\dagger}\right\}
\end{aligned}
$$

there is a delicate point. After taking the derivatives, one should not use such properties of the trace as cyclic permutations, since the sums in question do not converge absolutely. Disregarding this, one would miss total derivatives and hence an important part of the result. Consequently, we first get the function $\tilde{U}(\vec{q}, t)$ corresponding to $U_{p, p_{1}}$ etc. and then calculate

$$
\mathcal{L}_{k}=\int \frac{d^{2} q}{2 \pi}\left\{\tilde{U}(-\vec{q}, t) i \partial_{t} \tilde{U}^{\dagger}(\vec{q}, t)+(U \rightarrow V)\right\} .
$$

Using again the composition rule Eq. (20) in [18] we find up to fourth order in gradients $\mathcal{L}_{k}=\mathcal{L}_{k}^{0}+\mathcal{L}_{k}^{\text {top }}$ (a spurious imaginary term in $\mathcal{L}_{k}$ turns out to be the time derivative of the topological charge and hence vanishes [22]),

$$
\mathcal{L}_{k}^{0}=\frac{1}{4 \pi} \int d^{2} r\left[\partial_{t} \bar{\psi}(\vec{r}, t)+\cos \bar{\theta}(\vec{r}, t) \partial_{t} \bar{\phi}(\vec{r}, t)\right]
$$

and

$\mathcal{L}_{k}^{t o p}=\frac{-1}{16 \pi} \int d^{2} r\left\{\frac{\partial(\bar{\psi}, \cos \bar{\theta}, \bar{\phi})}{\partial(t, x, y)}-\partial_{t}\left[\cos \bar{\theta} \frac{\partial(\bar{\phi}, \bar{\psi})}{\partial(x, y)}\right]\right\}$.

Only the part $\mathcal{L}_{k}^{0}$ of $\mathcal{L}_{k}$ contributes to the equations of motion, since $\mathcal{L}_{k}^{\text {top }}$ is a total derivative in the action. As expected, we find from $\mathcal{L}_{k}^{0}$ the kinetics of a system of spins $\frac{1}{2} \cdot \mathcal{L}_{k}^{0}$ can be expressed in several ways; here it is given in a form explicitely depending on our representation of the unit vector $\vec{n}$

$$
\vec{n} \equiv(\sin \bar{\theta} \cos \bar{\phi}, \sin \bar{\theta} \sin \bar{\phi}, \cos \bar{\theta})
$$

which describes the spin in terms of the renormalized angles $\bar{\theta}$ and $\bar{\phi}$ cf. [23]. The $\bar{\psi}$ degree of freedom decouples in this part of the action. Since it also does not enter the definition of the spin- and charge-density, cf. [18], one would have a model with symmetry $S U(2) / U(1)$ for $\vec{n}$, if it were not for the second part $\mathcal{L}_{k}^{\text {top }}$, which we derived here for the first time.

$\mathcal{L}_{k}^{t o p}$ contains firstly a non-zero "Hopf term" and also an additional total derivative in time. The Hopf term is the linking coefficient of a smooth map $S_{3} \rightarrow S_{2}$ [24. Usually, it is expressed as [21]

$$
\mathcal{H}_{H o p f}=\frac{\Theta}{4 \pi^{2}} \int d^{2} r d t \epsilon^{\mu \nu \lambda}\left(z^{*} \partial_{\mu} z\right)\left(\partial_{\nu} z^{*} \partial_{\lambda} z\right) .
$$

Taking for the spinor $z$

$$
z=\left(e^{\frac{i}{2}(\bar{\psi}-\bar{\phi})} \cos \frac{\bar{\theta}}{2}, e^{\frac{i}{2}(\bar{\psi}+\bar{\phi})} \sin \frac{\bar{\theta}}{2}\right),
$$

then the unit vector $\vec{n}=z^{*} \vec{\sigma} z$ ( $\sigma^{j}$ are the Pauli matrices), and we identify $\mathcal{H}_{\text {Hopf }}$ with the first term in brackets of our result (14). Its prefactor is such that the $\Theta$-angle describing the statistics of the Skyrmions in Ref. [20] is equal to $\pi$. Thus, in the language of [20], the skyrmions carry spin $\frac{1}{2}$. The microscopic derivation of this is the first of the main results of the present paper. The additional term in (14) finds a quite natural explanation: it enters in such a way that this part of the density of the effective Lagrangian becomes a total spatial gradient (still including a time derivative) without any total time derivative.

Hamiltonian part Next we proceed to calculate the second part in the effective Lagrangian (10). Using the methods described above, $\tilde{S}^{j}(\vec{q})$ and $\tilde{N}(\vec{q})=2 \pi \delta(\vec{q})+$ $\delta N(\vec{q})$ are expanded in gradients of the fields and also $E(q)$ is expanded in $q$ up to fourth order. (We omit the time-argument which is unnecessary in this part.) In lowest order, the spin-density in real space is given by

$$
\tilde{S}^{j}(\vec{r})=\frac{1}{4 \pi} n^{j}(\vec{r}) .
$$

From $\vec{n}$, the charge-density is determined as

$$
\delta N(\vec{r})=\frac{1}{4 \pi} \vec{n}(\vec{r}) \cdot \partial_{x} \vec{n}(\vec{r}) \times \partial_{y} \vec{n}(\vec{r}) .
$$

The next order in the gradient expansion, i.e. second for $\tilde{S}^{j}$ and fourth for $\tilde{N}$ vanishes and one is left with the calculation of the fourth order for $\tilde{S}^{j}$. Collecting all terms together, we find $<\Psi_{H F}|\hat{H}| \Psi_{H F}>=\mathcal{L}_{H}+\mathcal{O}\left(\nabla^{6}\right)$ [22]

$$
\begin{aligned}
\mathcal{L}_{H} & =\frac{E(0)}{8 \pi} \int d^{2} r\left\{\frac{1}{4} \sum_{\alpha=x, y}\left(\partial_{\alpha} \vec{n}\right)^{2}-\vec{n} \cdot \partial_{x} \vec{n} \times \partial_{y} \vec{n}\right\} \\
& -\frac{3 E(0)}{2^{9} \pi} \int d^{2} r(\triangle \vec{n})^{2}+\frac{1}{2} \sum_{\vec{q}} \tilde{V}(q)|\delta N(\vec{q})|^{2} .
\end{aligned}
$$

Eq. (20) is the second main result of the present paper. The terms of second order in derivatives in (20) represent a $O(3)$ non-linear sigma model with topological term with a real prefactor. For a discussion of these second order terms in (20), cf. [18]. As regards the next order, one could have written down a number of terms of fourth order in derivatives of the components of $\vec{n}$ which 
all show the required symmetries. Surprisingly however, apart from the Coulomb energy which had to come out, there is basically no correction to the lower order calculation in which the Skyrmions are still non-interacting. One gets only the term proportional to $\triangle^{2}$ which comes from the direct expansion of $E(q)$ (its numerical prefactor is here given for the Coulomb interaction and the lowest Landau level). This term is only a small correction in our expansion in the variation of the fields on the scale of the magnetic length. A simple estimate shows that it contributes a term of the order of $-\frac{e^{2}}{\kappa l_{H}}\left(l_{H} / r_{0}\right)^{4}\left(r_{0}\right.$ is the size of the Skyrmion) to the energy of a single Skyrmion and thus does not help to stabilize the Skyrmion for small values of the $g$-factor. In (20), a total derivative in spatial coordinates was omitted which in all cases we checked was non-singular and thus did not contribute to (20).

We thank M. Potemski for sharing the results of 13 with us prior to publication. One of us (Yu.A. B.) thanks the PTB for hospitality and acknowledges support of the INTAS-94-4055 and the RFFI 95-02-05745 projects.

[1] Yu.A. Bychkov, S.V. Iordanskii, and G.M. Eliashberg, Pis'ma Zh.Eksp.Teor.Fiz., 33, 152 (1981) [JETP Lett. 33, 143 (1981)].

[2] C. Kallin and B.I. Halperin, Phys. Rev. B30, 5655 (1984).

[3] R.J. Nicholas, R.J. Haug, K. v.Klitzing, and G. Weimann, Phys. Rev. B37, 1294 (1988).

[4] A. Usher, R.J. Nicholas, J.J. Harris, and C.T. Foxon, Phys. Rev. B41, 1129 (1990).

[5] S.L. Sondhi, A. Karlhede, S.A. Kivelson, and E.H. Rezayi, Phys. Rev. B47, 16419 (1993).

[6] H.A. Fertig, L. Brey, R. Cote, and A.H. MacDonald, Phys. Rev. B50, 11018 (1994).

[7] A.A. Belavin and A.M. Polyakov, Pis'ma Zh.Eksp.Teor.Fiz., 22, 503 (1975) [JETP Lett. 22, 245 (1975)].

[8] R. Rajaraman, Solitons and Instantons (North-Holland, Amsterdam, 1989).

[9] E. Fradkin, Field theories of condensed matter systems (Addison-Wesley, Reading MA, 1990).

[10] S.E. Barret, G. Dabbagh, L.N. Pfeifer, K.W. West and R. Tycko, Phys. Rev. Lett. 74, 5112 (1995).

[11] A. Schmeller, J.P. Eisenstein, L.N. Pfeiffer, and K.W. West, Phys. Rev. Lett. 75, 4290 (1995).

[12] E.H. Aifer, B.B. Goldberg, and D.A. Broido, Phys. Rev. Lett. 76, 680 (1996).

[13] D.K. Maude, M. Potemski, J.C. Portal et al. (unpublished).

[14] K. Moon, H. Mori, Kun Yang, S.M. Girvin, and A.H. MacDonald, Phys. Rev. B51, 5138 (1995).

[15] L.Brey, H.A. Fertig, R. Cote, and A.H. MacDonald,
Phys. Rev. Lett, 75, 2562 (1995).

[16] N. Read and S. Sachdev, Phys. Rev. Lett. 75, 3509 (1996).

[17] M. Kasner and A.H. MacDonald, Phys. Rev. Lett. 76, 3204 (1996).

[18] Yu.A. Bychkov, T. Maniv, and I.D. Vagner, Phys. Rev. B53, 10148 (1996).

[19] I. Affleck in Fields, Strings and Critical Phenomena, edited by E. Brézin and J. Zinn-Justin, Les Houches Session 1988 XLIX (North-Holland, Amsterdam, 1990), p. 563.

[20] F. Wilczek and A. Zee, Phys. Rev. Lett. 51, 2250 (1983).

[21] Yong-shi Wu and A. Zee, Phys. Lett. 147B, 325 (1984).

[22] A detailed account of this work will be published later.

[23] J.R. Klauder, Phys. Rev. D19, 2349 (1979).

[24] B.A. Dubrovin, S.R. Novikov, and A.T. Fomenko, Contemporary Geometry (Springer-Verlag, New-York, 1986), Vol. 2. 